Revista de Comunicación y Salud, 2021 Vol. 11, 125-141

Editado por Cátedra de Comunicación y Salud

ISSN: 2173-1675

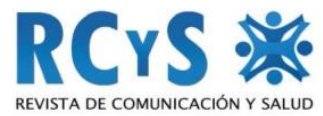

Enviado: 08/03/2021

Aprobado 06/05/2021

Publicado 27/05/2021

\title{
SOBRE LA PUBLICIDAD DE LOS MEDICAMENTOS: LEGISLACIÓN
}

\author{
About Advertising of Medicines: Legislation \\ Carlos del Castillo Rodríguez \\ Universidad Complutense de Madrid. España. \\ carlosdelcastillo@farm.ucm.es \\ Silvia Enríquez Fernández ${ }^{1}$ \\ Universidad Complutense de Madrid. España. \\ silvienr@ucm.es
}

\section{Cómo citar el artículo}

Castillo Rodríguez, C., y Enríquez Fernández, S. (2021). Sobre la publicidad de los medicamentos: legislación. Revista de Comunicación y Salud, 11, 125-141. https://doi.org/10.35669/rcys.2021.11.e276

\section{Resumen}

La publicidad de los medicamentos está sometida a un régimen especial justificado por los posibles efectos negativos que su práctica puede originar en la sociedad actual y consecuentemente en la salud pública. Las principales normas específicas que regulan el tema objeto de estudio son, entre otros, el Real Decreto Legislativo 1/2015, de 24 de julio, por el que se aprueba el texto refundido de la Ley de garantías y uso racional de los medicamentos y productos sanitarios y el Real Decreto 1416/1994, de 25 de junio, por el que se regula la publicidad de los medicamentos de uso humano. En éstas se dispone tanto las características de la publicidad como el control al que estará sometido y las infracciones imponibles en caso de incumplimiento.

Palabras clave: Legislación; publicidad; medicamentos; infracciones; intervención; administración pública.

\footnotetext{
${ }^{1}$ Silvia Enríquez Fernández: Graduada en Farmacia, Universidad Complutense de Madrid; Investigadora doctoral de la Universidad Complutense de Madrid. Departamento de Farmacia Galénica y Tecnología Alimentaria (Historia de la Farmacia y Legislación Farmacéutica). Contacto: silvienr@ucm.es.
} 


\begin{abstract}
The publicity of medicines is subject to a special regime which is justified by the importance of negative effects and the risk it may pose to public health. The specific rules governing the subject under consideration are in the Royal Legislative Decree 1/2015 of 24 July approving the consolidated text of the Law on Guarantees and Rational Use of Medicines and Medical Products and in the Royal Decree 1416/1994 of 25 June regulating the advertising of medicines for human use. These normative provide the characteristics of the advertising and the control to which it will be subject and the taxable infringements in case.
\end{abstract}

Keywords: Legislation; publicity; medicines; control; infringements; intervention; Public administration.

\title{
1. CONCEPTO DE PUBLICIDAD: PUBLICIDAD DE MEDICAMENTOS
}

La definición general de publicidad está establecida en la Ley 34/1988, de 11 de noviembre, General de Publicidad ${ }^{2}$. Su artículo segundo define la publicidad como: «toda forma de comunicación realizada por una persona física o jurídica, pública o privada, en el ejercicio de una actividad comercial, industrial, artesanal o profesional, con el fin de promover de forma directa o indirecta la contratación de bienes muebles o inmuebles, servicios, derechos y obligaciones».

La única referencia explícita que encontramos a los medicamentos en la mencionada Ley se encuentra en el artículo 5.4 el cuál dispone que: «los productos estupefacientes, psicótropos y medicamentos, destinados al consumo de personas, solamente podrán ser objeto de publicidad en los casos, formas y condiciones establecidos en las normas especiales que los regulen». Dicha norma nos remite a la regulación específica para la publicidad de medicamentos.

La normativa especial sobre publicidad es muy extensa, y la podemos clasificar en tres grupos según la información que nos ofrece: en función del medio de difusión, en función de los destinatarios de la publicidad, y en función del producto. Las normas relativas a la publicidad de los medicamentos se encuadran en el último de estos grupos ${ }^{3}$, es decir, incluimos los medicamentos dentro de la legislación publicitaria en función del producto.

El mercado de los medicamentos es diverso al de otros productos, por ello está sometido a un régimen especial el cuál se justifica por la predominancia de la protección de los intereses de los consumidores, así como el daño que puede causar a la salud

${ }^{2}$ BOE núm. 274 de 15 de noviembre de 1988.

${ }^{3}$ Ibíd. 
pública frente a los intereses del anunciante ${ }^{4}$. Por ello existe una regulación específica que lo que pretende es que este tipo de publicidad sea verdadera e informativa ${ }^{5}$.

La primera regulación específica publicada en el territorio nacional sobre publicidad de medicamentos fue la Orden de 10 de diciembre de 1985, del Ministerio de Sanidad y Consumo, por la que se regulan los mensajes publicitarios referidos a medicamentos y determinados productos sanitarios ${ }^{6}$, reguló el contenido de los mensajes publicitarios de todo tipo dirigidos al público en general y referidos a medicamentos. Posteriormente, en el año 1994, se publicó el Real Decreto 1416/1994, de 25 de junio, por el que se regula la publicidad de medicamentos de uso humano ${ }^{7}$, sobre la cuál trataremos en el apartado III. Así también, en el Real Decreto Legislativo 1/2015, de 24 de julio, por el que se aprueba el texto refundido de la Ley de garantías y uso racional de los medicamentos y productos sanitarios ${ }^{8}$, a la que se hará referencia a continuación, se regula específicamente el tema objeto de estudio.

El concepto legal de publicidad de medicamentos -regulado en esta normativa específica- es el mismo en el ámbito nacional ${ }^{9}$ que en el ámbito del Derecho comunitario $^{10}$, y ambos definen como publicidad de medicamentos toda forma de oferta informativa, de prospección o de incitación destinada a promover la prescripción, la dispensación, la venta o el consumo de medicamentos. Este concepto comprende la publicidad de medicamentos destinada al público, así como también aquella destinada a personas facultadas para prescribirlos o dispensarlos, como es, el patrocinio de reuniones promocionales; el suministro de muestras; la actuación de los visitadores médicos; la incitación a prescribir o dispensar medicamentos mediante concesión, oferta o promesa de ventajas, pecuniarias o en especie excepto cuando su valor intrínseco resulte mínimo; el patrocinio de congresos científicos en los que participen personas facultadas para prescribir o dispensar medicamentos y, en particular, el hecho de correr a cargo con los gastos de desplazamiento y estancia con motivo de dichos congresos.

\footnotetext{
${ }^{4}$ Su régimen jurídico es especial y particular dado su íntima relación con la protección de la salud. Sobre este tema existe mucha literatura crítica. Sobre este tema, véase: BOMBILLAR SÁENZ, Francisco Miguel (2010). Intervención administrativa y régimen jurídico del medicamento en la Unión Europea. Tesis Doctoral. Granada: Ed. Universidad de Granada; L. SARRATO MARTíneZ. (2015). Régimen jurídicoadministrativo del medicamento. Las Rozas (Madrid): La Ley. J. VIDA FERNÁNDEZ. Concepto y régimen jurídico de los medicamentos. Su distinción con otros productos para el cuidado de la salud. Valencia: Ed. Tirant lo Blanch. J. VIDA Fernández; J. Faus SANTASusana, [dirs.]. (2017). Tratado de Derecho Farmacéutico. Cizur Menor (Navarra): Ed. Thomson Reuters-Aranzadi

${ }^{5}$ Ministerio de Sanidad, Consumo y Bienestar Social. 2019. Guía para la publicidad de medicamentos de uso humano dirigida al público, cit. p. 5.

${ }^{6}$ BOE núm. 302, de 18 de diciembre de 1985.

${ }^{7}$ BOE núm. 180, de 29 de julio de 1994.

${ }^{8}$ BOE núm. 177, de 25 de julio de 2015. Realizaremos un apartado específico sobre esta ley.

'Véase, artículo 1.2. BOE núm. 180, de 29 de julio de 1994. Real Decreto 1416/1994, de 25 de junio, por el que se regula la publicidad de los medicamentos de uso humano.

10 Véase, artículo 86. DOCE. L 311. 6 de noviembre de 2001. Directiva 2001/83/CE, del Parlamento Europeo y del Consejo, de 6 de noviembre de 2001, por la que se establece un código comunitario sobre medicamentos de uso humano
} 
Sobre la publicidad de los medicamentos: legislación

\section{OBJETIVOS}

El objetivo del presente artículo es realizar un estudio exhaustivo acerca del régimen jurídico de la publicidad sobre los medicamentos y analizar la actuación de las diferentes Administraciones públicas en la materia.

\section{NORMATIVA APLICABLE A LA PUBLICIDAD DE MEDICAMENTOS}

\subsection{Normativa comunitaria}

En el marco legal comunitario europeo, las directivas que hacen referencia o afectan a la regulación de la publicidad de los medicamentos son (ordenadas cronológicamente):

- La Directiva 2001/83/CE, del Parlamento Europeo y del Consejo, de 6 de noviembre de 2001, por la que se establece un código comunitario sobre medicamentos de uso humano ${ }^{11}$, que como hemos mencionado define el concepto de publicidad de medicamentos en su artículo 86. Además, en los artículos 87 a 90 , se define cuáles son aquellos medicamentos cuya publicidad está totalmente prohibida y los requisitos que tendrá que cumplir en aquellos los cuales sí esté autorizada ${ }^{12}$. En los artículos 97 a 100 responsabiliza de la publicidad al titular de la comercialización del medicamento, otorgando a los tribunales y a los órganos administrativos de los Estados miembros la capacidad de exigir una publicidad que se ajuste a la normativa vigente.

- La Directiva 2006/114/CE, del Parlamento Europeo y del Consejo, de 12 de diciembre de 2006, sobre publicidad engañosa y publicidad comparativa ${ }^{13}$.

- Directiva 2010/13/UE, del Parlamento Europeo y del Consejo, de 10 de marzo de 2010, sobre la coordinación de determinadas disposiciones legales, reglamentarias y administrativas de los Estados miembros relativas a la prestación de servicios de comunicación audiovisual ${ }^{14}$.

\footnotetext{
${ }^{11}$ Diario Oficial de las Comunidades Europeas. L 311. 6 de noviembre de 2001.

12 Véase, los artículos 87 a 90 de la mencionada Directiva (DOE 311 de 6 de noviembre de 2001) en los cuáles se establece las condiciones que debe contener la publicidad de un medicamento. En virtud de su importancia vamos a destacar que la publicidad de un medicamento deberá favorecer la utilización racional del mismo, presentándolo de forma objetiva y sin exagerar sus propiedades. En estos artículos también se hace referencia a prohibiciones que se establecen en los Estados miembros como mencionar en aquella publicidad destinada al público, de indicaciones terapéuticas tales como: la tuberculosis, las enfermedades de transmisión sexual, otras enfermedades infecciosas graves, el cáncer y otras enfermedades tumorales, el insomnio crónico, y la diabetes y otras enfermedades del metabolismo.

13 DOCE, L 376 de 12 de diciembre de 2006. La mencionada directiva no hace referencia a los medicamentos concretamente, sin embargo, regula las características de la publicidad engañosa, la cuál esta prohibida por el artículo 87 de la Directiva 2001/83/CE, del Parlamento Europeo y del Consejo, de 6 de noviembre de 2001, por la que se establece un código comunitario sobre medicamentos de uso humano. Según la primera, publicidad engañosa es toda publicidad que, de una manera cualquiera, incluida su presentación, induce a error o puede inducir a error a las personas a las que se dirige o afecta y que, debido a su carácter engañoso, puede afectar su comportamiento económico o que, por estas razones, perjudica o es capaz de perjudicar a un competidor.

${ }^{14}$ DOCE, L 75 de 10 de marzo de 2010. Vid. Artículo 10.3 que restringe las condiciones de promoción y prohíbe la publicidad de medicamentos sujetos a prescripción médica, el articulo $11.4 \mathrm{~b}$ por el que se prohíbe a los programas no podrán colocar medicamentos y artículo 21 por el cuál queda prohibida la
} 


\subsection{Normativa en el ámbito nacional}

Con respecto a la normativa nacional que afecta a la publicidad de medicamentos, ordenados cronológicamente encontramos:

- La Orden de 10 de diciembre de 1985, del Ministerio de Sanidad y Consumo, por la que se regulan los mensajes publicitarios referidos a medicamentos y determinados productos sanitarios ${ }^{15}$.

- La Ley 14/1986, de 26 de abril, General de Sanidad (LGS, añadir abreviatura) ${ }^{16}$. En el artículo 27 hace referencia a la publicidad sanitaria abordando el control de la veracidad de la publicidad y propaganda comerciales en materia de salud. Dicho artículo establece que las Administraciones públicas realizarán un control de la publicidad y propaganda comercial para que se ajusten a criterios de veracidad, en el ámbito de sus competencias para limitar todo aquello que pueda constituir un perjuicio para la salud. Este artículo fue modificado por la Ley 33/2011, General de Salud Pública ${ }^{17}$, la cuál mantiene lo promulgado por la LGS, pero añade a la misma «con especial atención a la protección de la salud de la población más vulnerable».

- La Ley 34/1988, de 11 de noviembre, General de Publicidad, como hemos mencionado, hace referencia al concepto de publicidad de medicamentos, y para su regulación refiere a la legislación especifica sobre éstos.

- La Ley 3/1991, de 10 de enero, de Competencia Deslea/18, establece la prohibición de los actos de competencia desleal, incluida la publicidad ilícita en los términos de la Ley General de Publicidad.

- El Real Decreto 1416/1994, de 25 de junio, por el que se regula la publicidad de los medicamentos de uso humano ${ }^{19}$.

- La Circular 6/95, de la Dirección General de Farmacia y Productos Sanitarios del Ministerio de Sanidad y Consumo, aclaraciones al Real Decreto 1416/1994, de 25 de junio, por la que se regula la publicidad de los medicamentos de uso humano, modificada por la Circular 7/99, de 27 de mayo, de la Dirección General de Farmacia y Productos Sanitarios ${ }^{20}$.

- El Real Decreto 1345/2007, de 11 de octubre, por el que se regula el procedimiento de autorización, registro y condiciones de dispensación de los medicamentos de uso humano fabricados industrialmente, en el artículo 25, establece tres requisitos

televenta de medicamentos sujetos a una autorización de comercialización en el sentido de la Directiva 2001/83/CE, así como la televenta de tratamientos médicos.

${ }^{15}$ BOE núm. 302, de 18 de diciembre de 1985.

${ }^{16}$ BOE núm. 102 de 29 de abril de 1986.

${ }^{14}$ BOE núm. 240, de 05 de octubre de 2011.

${ }^{18}$ BOE núm. 10, de 11 de enero 1991.

${ }^{19}$ BOE núm. 180, de 29 de julio de 1994. A continuación, analizaremos el contenido específico de este Real Decreto.

${ }^{20}$ En la mencionada circular se realizan aclaraciones al Real Decreto 1416/94, de 25 de junio, por el que se regula la publicidad de los medicamentos de uso humano. 
que deberán cumplir los medicamentos cuya publicidad este destinada al público ${ }^{21}$.

- La Ley 29/2009, de 30 de diciembre, por la que se modifica el régimen legal de la competencia desleal y de la publicidad para la mejora de la protección de los consumidores y usuarios ${ }^{22}$.

- El Real Decreto Legislativo 1/2015, de 24 de julio, por el que se aprueba el texto refundido de la Ley de garantías y uso racional de los medicamentos y productos sanitarios $^{23}$.

\section{REGULACIÓN ESPECIFICA: REAL DECRETO 1416/1994, DE 25 DE JUNIO, POR EL QUE SE REGULA LA PUBLICIDAD DE LOS MEDICAMENTOS DE USO HUMANO}

Las disposiciones incluidas en el Real Decreto 1416/1994, de 25 de junio, por el que se regula la publicidad de los medicamentos de uso humano ${ }^{24}$, se aplican a la publicidad de medicamentos de uso humano fabricados industrialmente.

Podríamos resumir los principios de este Real Decreto en cuatro. En primer lugar, se prohíbe la publicidad de un medicamento que no haya obtenido la correspondiente autorización de comercialización. En segundo lugar, todos los elementos de la publicidad de un medicamento tienen que ajustarse a la información que figura en la ficha técnica del mismo. En tercer lugar, la publicidad siempre tiene que favorecer el uso racional del medicamento presentándolo de forma objetiva y sin exagerar sus propiedades. Por ultimo, prohíbe la publicidad engañosa mencionando el artículo 4 de la Ley 34/1988, de 11 de noviembre, General de Publicidad, sobre la prohibición de la publicidad subliminal25.

La regulación en esta ley se hace mediante la distinción de dos tipos de publicidad: la destinada al público y la destinada a las personas facultadas para prescribir. En virtud de la importancia de ambos tipos de publicidad, veremos como se regulan.

\subsection{Publicidad destinada al público}

Se entenderá por publicidad de medicamentos destinada al público en general, aquélla que, habiendo sido debidamente autorizada, se dirija al público con fines promocionales

${ }^{21}$ Véase, BOE núm. 267, de 7 de noviembre de 2007. Los tres requisitos que se establecen en el mencionado artículo son: 1. Que no se financien con fondos públicos. 2. Que por su composición y objetivo estén concebidos y destinados para ser utilizados sin la intervención de un médico que realice el diagnóstico, la prescripción o el seguimiento del tratamiento, y que no contengan en su composición sustancias psicotrópicas ni estupefacientes.

22 BOE núm. 315, de 31 de diciembre de 2009.

${ }^{23}$ BOE núm. 177, de 25 de julio de 2015. Realizaremos un apartado específico sobre esta ley.

${ }^{24}$ BOE núm. 180, de 29 de julio de 1994.

${ }^{25}$ BOE núm. 274 de 15 de noviembre de 1988. Ley 34/1988, de 11 de noviembre, General de Publicidad. Artículo 4, sobre la publicidad subliminal, «a los efectos de esta ley, será publicidad subliminal la que mediante técnicas de producción de estímulos de intensidades fronterizas con los umbrales de los sentidos o análogas, pueda actuar sobre el público destinatario sin ser conscientemente percibida». 
e informativos siempre promoviendo el uso adecuado del medicamento ${ }^{26}$. En la misma, deberá observarse el carácter publicitario de forma evidente, además de contener datos identificativos del medicamento y las recomendaciones que se determinen por el Ministerio con competencia en sanidad para prevenir los riesgos derivados de la utilización normal de los mismos.

Con respecto a la información que deberá incluir como mínimo la publicidad objeto de estudio es la denominación del medicamento ${ }^{27}$, las informaciones indispensables para promover su utilización racional y una invitación expresa a leer detenidamente las instrucciones que contiene el medicamento.

Cabe destacar que la publicidad de un medicamento destinada al público podrá incluir solamente la denominación del mismo cuando su único objetivo sea recordar dicha denominación, siempre que dicho medicamento sea lo suficientemente conocido por el público y haya permanecido en campañas promocionales, al menos, durante dos años. En el mensaje publicitario aparecerá la mención: «en caso de duda consulte a su farmacéutico» o una expresión similar.

La publicidad de un medicamento destinado al público no podrá incluir ningún elemento que 28 :

a) Se atribuya a la consulta médica o a la intervención quirúrgica un carácter innecesario, ofreciendo un diagnóstico o aconsejando un tratamiento por correspondencia.

b) Sugiera que su efecto está asegurado, que no tiene efectos secundarios o que sus efectos son superiores o iguales al de otro tratamiento o medicamento.

c) Sugiera que el usuario puede mejorar su salud gracias a él o que ésta puede verse afectada en caso de su no utilización ${ }^{29}$.

d) Sugiera o indique que su uso aumenta el rendimiento deportivo.

e) Se dirija, exclusiva o principalmente, a niños.

f) Se refiera a una recomendación que hayan formulado profesionales de la salud $u$ otras personas que puedan por su posición o prestigio incitar al consumo de medicamentos.

g) Haga una comparación del medicamento con un producto alimenticio, cosmético o cualquier otro producto de consumo.

h) Sugiera que la seguridad o la eficacia del medicamento están producidas por una sustancia natural.

\footnotetext{
${ }^{26}$ Véase, articulo 22. BOE núm. 180, de 29 de julio de 1994, sobre la autorización de la publicidad destinada al público.

27 Véase, articulo 5. BOE núm. 180, de 29 de julio de 1994, deberá incluir la denominación del medicamento, así como la Denominación Oficial Española o en su defecto la Denominación Común Internacional, o la denominación común usual o científica cuando el medicamento contenga un único principio activo.

${ }^{28}$ Véase, articulo 6 sobre prohibiciones. BOE núm. 180, de 29 de julio de 1994

${ }^{29}$ Esta prohibición no se aplicará a las campañas de vacunación reguladas en el artículo 9 de este Real Decreto sobre campañas de vacunación.
} 
i) Pueda incitar a la realización de un falso autodiagnóstico, bien por una descripción o representación detallada de la anamnesis.

j) Se refiera a testimonios de curación de forma abusiva, alarmante o engañosa.

k) Utilice representaciones visuales de las alteraciones del cuerpo humano producidas por enfermedades, lesiones, o la acción de un medicamento en el mismo.

I) Mencione características obvias del medicamento, como es que, el medicamento ha recibido la autorización sanitaria o cualquier otra autorización.

\subsection{Publicidad destinada a profesionales sanitarios}

La información que obtienen los profesionales sanitarios sobre los medicamentos es uno de los factores que puede influir a la hora de que estos prescriban un medicamento u otro, por eso resulta imprescindible que la publicidad que se hace de ellos este regulada por el Derecho. Este tipo de publicidad deberá contener la información completa para que los profesionales sanitarios puedan juzgar el valor terapéutico del medicamento. Para ello, el Real Decreto 1416/1994, de 25 de junio, por el que se regula la publicidad de los medicamentos de uso humano ${ }^{30}$, establece la información técnico-científica mínima que deberá contener la publicidad es la información esencial del producto según los datos contenidos en la ficha técnica ${ }^{31}$, su régimen de prescripción y dispensación y las diferentes presentaciones del producto, y la dosificación y la forma farmacéutica.

Existen distintas formas de realizar publicidad destinada a los profesionales sanitarios facultados para prescribir o dispensar medicamentos. Una de las formas es la visita médica, por la cuál un visitador médico acude a los profesionales sanitarios para transmitirles conocimientos técnicos sobre el medicamento objeto de la publicidad. Este tipo de publicidad requiere que los visitadores médicos reciban la formación adecuada por parte del laboratorio que comercia el citado medicamento. En cada visita se incluirá el precio de venta al público, las condiciones de la presentación farmacéutica que financia el Sistema Nacional de Salud, en su caso, y, cuando sea posible, la estimación del coste del tratamiento ${ }^{32}$. Asimismo, en cada visita médica proporcionarán una ficha técnica al profesional sanitario sobre el medicamento publicitado. Por último, éstos notificarán al servicio científico ${ }^{33}$ toda la información que reciban de los profesionales sanitarios visitados.

Otro tipo de publicidad destinada a profesionales sanitarios facultados para prescribir o dispensar es la denominada «publicidad documental». Esta se realiza a través de

\footnotetext{
${ }^{30}$ Véase, artículo 10. BOE núm. 180, de 29 de julio de 1994.

${ }^{31}$ Deberá incluir al menos: nombre del medicamento, composición cualitativa y cuantitativa, datos clínicos completos, incompatibilidades, instrucciones de uso/manipulación, nombre y dirección del titular de la autorización.

32 Véase, artículo 12.3. BOE núm. 180, de 29 de julio de 1994

${ }^{33}$ Véase, artículo 20. BOE núm. 180, de 29 de julio de 1994, según el cuál «el titular de la autorización de un medicamento contará con un servicio científico dentro de su empresa encargado de la información relativa a los medicamentos que ponga en el mercado».
} 
publicaciones como revistas, boletines, libros y similares (de carácter científico), o incorporada a medios audiovisuales en soporte óptico, o similar, siempre y cuando estén dirigidas exclusivamente a personas facultadas para prescribir 0 dispensar medicamentos.

También se puede realizar publicidad por medio de muestras gratuitas. Estas se realizarán exclusivamente a las personas facultadas para prescribir medicamentos, se podrán distribuir un máximo de diez muestras de cada medicamento por año y persona facultativa, y durante un tiempo máximo de dos años los cuáles comienzan a contabilizarse desde la fecha de autorización del medicamento.

Por último, cabe mencionar otros dos tipos de actos publicitarios, por un lado, los incentivos $^{34}$ los cuáles están prohibidos, y, por otro lado, el patrocinio de reuniones científicas. Estas estarán permitidas, en ciertos casos, siempre y cuando sus destinatarios sean facultativos en ejercicio clínico o las entidades en que se asocian.

\section{REGULACIÓN ESPECIFICA INCLUIDA EN EL REAL DECRETO LEGISLATIVO 1/2015, DE 24 DE JULIO, POR EL QUE SE APRUEBA EL TEXTO REFUNDIDO DE LA LEY DE GARANTÍAS Y USO RACIONAL DE LOS MEDICAMENTOS Y PRODUCTOS SANITARIOS}

El Real Decreto Legislativo $1 / 2015^{35}$ incluye algunas especificaciones sobre el tema objeto de estudio en este trabajo, concretamente su artículo 80. En el primer apartado del citado artículo, establece los tres requisitos que se deberán cumplir para poder publicitar un medicamento, estos también se encuentran recogidos en el Real Decreto $1345 / 2007^{36}$. En primer lugar, solo se podrá realizar publicidad de aquellos medicamentos que no se financien con fondos públicos. En segundo lugar, de aquellos que, estén destinados para un uso sin la intervención de un médico que realice el diagnóstico, la prescripción o el seguimiento del tratamiento, aunque requieran la intervención de un farmacéutico. Este requisito podrá exceptuarse cuando se realicen campañas de vacunación aprobadas por las autoridades sanitarias competentes. En tercer y último lugar, no constituyan sustancias psicotrópicas o estupefacientes con arreglo a lo definido en los convenios internacionales ${ }^{37}$.

\footnotetext{
${ }^{34}$ Véase, artículo 17, BOE núm. 180, de 29 de julio de 1994 por el cuál: «queda prohibido otorgar, ofrecer o prometer a las personas facultadas para prescribir o dispensar medicamentos y en el marco de la promoción de los mismos frente a dichas personas, primas, ventajas pecuniarias o ventajas en especie, con excepción de aquellas que tengan un valor insignificante y que sean irrelevante para la práctica de la medicina o la farmacia». Tampoco podrán las personas facultadas para prescribir o dispensar medicamentos aceptar ninguno de los incentivos prohibidos que se disponen en el artículo 17 del Real Decreto objeto de estudio.

${ }^{35}$ BOE núm. 177, de 25 de julio de 2015.

${ }^{36}$ Véase, artículo 80.1. BOE núm. 177, de 25 de julio de 2015. Se hace una excepción no establecida en el segundo requisito del Real Decreto 1345/2007, «que por su composición y objetivo estén concebidos y destinados para ser utilizados sin la intervención de un médico que realice el diagnóstico, la prescripción o el seguimiento del tratamiento". En el Real Decreto Legislativo 1/2015 este requisito podrá exceptuarse cuando se realicen campañas de vacunación aprobadas por las autoridades sanitarias competentes.

37 Véase, artículo 80.1. BOE núm. 177, de 25 de julio de 2015.
} 
Cabe destacar el apartado 3 del artículo $80^{38}$, según el cuál la publicidad de medicamentos no sujetos a prescripción médica no requerirá de una autorización administrativa previa. El Ministerio de Sanidad, Consumo y Bienestar Social establecerá unos controles destinados a que se evalúe la publicidad dirigida al público para ellos (se explicarán en el apartado 6 sobre control y vigilancia de la publicidad sanitaria). Este artículo prohíbe las primas, obsequios, premios, concursos, bonificaciones o similares como métodos vinculados a la promoción o venta al público de estos medicamentos, en cualquier caso. En el resto de los apartados del mismo artículo se recogen prohibiciones especificas de medicamentos para los cuáles no se puede realizar publicidad destinada al público.

\section{CONTROL Y VIGILANCIA DE LA PUBLICIDAD DE MEDICAMENTOS}

La normativa comunitaria ha establecido un sistema de control sobre la promoción de medicamentos que se caracteriza por ser doble, interno y externo. En este sentido, por un lado, como mecanismo de control interno, las empresas responsables de la comercialización pueden contar con un servicio científico y velar así por el cumplimiento de la normativa en cuestión. Por otro lado, el control externo es ejercido por órganos tanto jurisdiccionales como administrativos, sin perjuicio del papel que en materia de publicidad juega la autorregulación ${ }^{39}$.

\subsection{Control interno}

Para realizar un control interno existen diversos medios. Por ejemplo, en España, existe una asociación sin ánimo de lucro denominada Asociación para la Autorregulación de la Comunicación Comercial (AUTOCONTROL). Dicha asociación está integrada por agencias de publicidad, anunciantes, asociaciones profesionales y medios de comunicación. Su objetivo es trabajar por una publicidad responsable: legal, veraz, leal y honesta ${ }^{40}$. Entre sus servicios encontramos consultas para analizar un proyecto antes de su emisión. En este sentido, resuelve a través del Jurado de la Publicidad las reclamaciones que se le plantean relacionadas con mensajes publicitarios dirigidos al público sobre medicamentos de uso humano ${ }^{41}$.

\footnotetext{
${ }^{38}$ Véase, artículo 80.1. BOE núm. 177, de 25 de julio de 2015. «la publicidad de medicamentos no sujetos a prescripción médica no requerirá de autorización administrativa previa, si bien las Administraciones sanitarias competentes efectuarán los controles necesarios para garantizar que los contenidos publicitarios cumplan con las normas legales y reglamentarias, que les sean de aplicación y que se ajusten fielmente a las condiciones científicas y técnicas recogidas en la autorización de comercialización».

${ }^{39}$ Á. García Vidal. La promoción de medicamentos dirigida a profesionales sanitarios. Estudio desde la perspectiva del Derecho Mercantil. Marcial Pons. Madrid. 2013. 271 págs.

${ }^{40}$ Véase, página web www.autocontrol.es.

41 Véase, Ministerio de Sanidad, Consumo y Bienestar Social. 2019. Guía para la publicidad de medicamentos de uso humano dirigida al público. p. 5.
} 


\subsection{Control externo}

En primer lugar, el control administrativo tiene su fundamento en el artículo 30 de la de la Ley 14/1986, de 26 de abril, General de Sanidad ${ }^{42}$, según la cuál «todos los centros y establecimientos sanitarios, así como las actividades de promoción y publicidad, estarán sometidos a la inspección y control por las Administraciones Sanitarias competentes». Asimismo, el artículo 5 de la Ley 34/1988, de 11 de noviembre, General de Publicidad en su primer apartado establece que «la publicidad de materiales o productos sanitarios y de aquellos otros sometidos a reglamentaciones técnico-sanitarias, así como la de los productos, bienes, actividades y servicios susceptibles de generar riesgos para la salud (...) podrá ser regulada por sus normas especiales o sometida al régimen de autorización administrativa previa. Dicho régimen podrá asimismo establecerse cuando la protección de los valores y derechos constitucionalmente reconocidos así lo requieran». Este artículo proporciona a las Administraciones la capacidad de controlar la publicidad antes de que se emita.

No obstante, se hará una mención a los controles que se realizan a la publicidad sobre medicamentos que no requieren autorización administrativa previa, los cuáles son aquellos que no están sujetos a prescripción médica ${ }^{43}$.

Estos controles los ejerce el Ministerio con competencias en sanidad en colaboración con otros organismos también implicados en este proceso, como, por ejemplo, la Asociación para la Autorregulación de la Comunicación Comercial, la cuál se ha mencionado anteriormente. Su finalidad ha sido la de asegurar que los mensajes publicitarios que se emitan cumplan con todas y cada una de las condiciones impuestas por el ordenamiento jurídico en esta materia, sin perjuicio del ejercicio de las competencias administrativas que puedan corresponder. Su objetivo principal será fomentar la autorregulación en materia de publicidad de medicamentos de uso humano dirigida al público en general ${ }^{44}$.

En segundo lugar, para iniciar una acción judicial en materia de publicidad de medicamentos, se recurre a la jurisdicción ordinaria mediante juicio verbal según lo establecido en el artículo 249.1.4 de la Ley 1/2000, de 7 de enero, de Enjuiciamiento

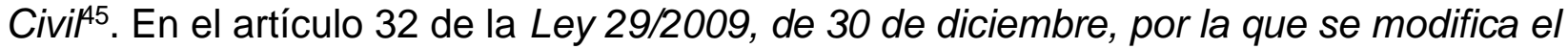
régimen legal de la competencia desleal y de la publicidad para la mejora de la protección de los consumidores y usuarios ${ }^{46}$ se regulan las diferentes acciones para llevar a cabo

\footnotetext{
42 BOE núm. 102 de 29 de abril de 1986.

${ }^{43}$ Véase, artículo 80.1. BOE núm. 177, de 25 de julio de 2015, según el cuál los medicamentos no sujetos a prescripción médica no requieren autorización administrativa previa.

44 Véase, Ministerio de Sanidad, Consumo y Bienestar Social. 2019. Guía para la publicidad de medicamentos de uso humano dirigida al público, p. 5.

${ }^{45}$ Véase, BOE núm. 7 de 8 de enero de 2000. Según el mencionado artículo: «se decidirán por el juicio ordinario cualquiera que sea su cuantía: las demandas relativas a publicidad siempre que no versen exclusivamente sobre reclamaciones de cantidad, en cuyo caso se tramitarán por el procedimiento que les corresponda en función de la cuantía que se reclame».

${ }^{46}$ BOE núm. 315, de 31 de diciembre de 2009.
} 
en los casos de publicidad ilícita: acción de cesación de la conducta desleal o de prohibición de su reiteración futura; acción de rectificación de las informaciones engañosas, incorrectas o falsas; acción declarativa de deslealtad; acción de remoción de los efectos producidos por la conducta desleal; acción de prohibición, si la conducta todavía no se ha puesto en práctica; acción de resarcimiento de los daños y perjuicios ocasionados por la conducta desleal, si ha intervenido dolo o culpa del agente; y, acción de enriquecimiento injusto.

Está legitimada para llevar a cabo estas acciones, cualquier persona física o jurídica que resulte afectada $y$, en general, quienes tengan un derecho subjetivo o un interés legítimo, asociaciones, corporaciones profesionales o representativas de intereses económicos, cuando resulten afectados los intereses de sus miembros. El control de la publicidad de medicamentos generalmente se realiza en defensa de los intereses generales, colectivos o difusos, de los consumidores y usuarios. De manera que también, gozaran de legitimación activa ${ }^{47}$ :

- El Instituto Nacional del Consumo y los órganos o entidades correspondientes de las Comunidades Autónomas y de las corporaciones locales competentes en materia de defensa de los consumidores y usuarios.

- Las asociaciones de consumidores y usuarios.

- Las entidades de otros Estados miembros de la Comunidad Europea constituidas para la protección de los intereses colectivos y de los intereses difusos de los consumidores y usuarios que estén habilitadas mediante su inclusión en la lista publicada a tal fin en el «Diario Oficial de la Unión Europea».

- El Ministerio Fiscal.

\section{INFRACCIONES TIPIFICADAS EN MATERIA DE PUBLICIDAD DE MEDICAMENTOS}

Las infracciones tipificadas en materia de publicidad en general, que se podrían aplicar en caso de publicidad ilícita de medicamentos se encuentran reguladas en diferentes normas.

En el artículo 5 de la Ley 34/1988, de 11 de noviembre, General de Publicidad ${ }^{48}$, apartado seis, se establece que el incumplimiento de las normas especiales que regulen la publicidad de los productos, bienes, actividades y servicios -entre los que encontramos la publicidad de medicamentos- tendrá consideración de infracción a los efectos previstos en el Real Decreto Legislativo 1/2007, de 16 de noviembre, por el que se aprueba el texto refundido de la Ley General para la Defensa de los Consumidores y Usuarios y otras leyes complementarias ${ }^{49}$ y en la Ley 14/1986, de 26 de abril, General de Sanidad ${ }^{50}$, en las cuáles se refieren a la normativa sobre publicidad especial.

\footnotetext{
47 Véase, artículo 33. BOE núm. 315, de 31 de diciembre de 2009.

${ }^{48}$ BOE núm. 274 de 15 de noviembre de 1988.

${ }^{49}$ BOE núm. 287, de 30 de noviembre de 2007. Véase artículos 49 a 52, en los cuáles vienen reguladas cuáles son las infracciones, así como los grados de infracción y sus respectivas sanciones según el grado.

${ }^{50}$ BOE núm. 102 de 29 de abril de 1986. Véase artículo 35 donde se tipifican las infracciones sanitarias.
} 
Con respecto a las infracciones especificas sobre publicidad de medicamentos, encontramos en el artículo 111 del Real Decreto Legislativo 1/2015, de 24 de julio, por el que se aprueba el texto refundido de la Ley de garantías y uso racional de los medicamentos y productos sanitarios ${ }^{51}$. Las infracciones se califican como leves, graves y muy graves atendiendo al riesgo para la salud, la gravedad de la alteración sanitaria y social producida, generalización de la infracción y reincidencia. Destacaremos algunas de ellas. El quinto apartado considera infracción leve realizar publicidad de fórmulas magistrales o de preparados oficinales. Entre las infracciones graves, el apartado 27 tipifica la conducta consistente en ofrecer directa o indirectamente incentivos, obsequios o primas, prohibidos por parte de quien tenga intereses directos o indirectos en la producción, fabricación y comercialización de medicamentos. Finalmente, como infracción muy grave se califica la conducta general consistente en realizar promoción, información o publicidad de medicamentos no autorizados o sin que tales actividades se ajusten a lo dispuesto en esta ley o en la legislación general sobre publicidad ${ }^{52}$. Para cada uno de los tipos de infracciones se fijan tres grados, ${ }^{53}$ y para cada uno de ellos un rango de multas imponibles ${ }^{54}$.

Por último, cabe destacar que, el Código Penal hace referencia a la publicidad de medicamentos. Por una parte, establece una pena de prisión de seis meses a un año o multa de 12 a 24 meses para aquella publicidad sobre medicamentos autorizados que puedan hacer alegaciones falsas o que manifiesten características inciertas ${ }^{55}$. Por otra parte, para aquellos medicamentos que no están autorizados o sea un producto perjudicial para la salud, se establece la pena de prisión de uno a cuatro años, multa de

\footnotetext{
51 BOE núm. 177, de 25 de julio de 2015.

52 Véase, Sánchez Ruiz, M.M. Capítulo noveno. Algunas cuestiones jurídicas sobre la publicidad de los medicamentos en Cuestiones actuales de la prestación farmacéutica y los medicamentos. Ma Belén García Romero y Mํㅡㄹ Del Mar de la Peña Amorós. 2017. ISBN: 978-84-9148-487-5.

${ }^{53}$ Véase, BOE núm. 177, de 25 de julio de 2015. Articulo 114. En función de la negligencia e intencionalidad del sujeto infractor, fraude, connivencia, incumplimiento de las advertencias previas, cifra de negocios de la empresa, número de personas afectadas, perjuicio causado, beneficios obtenidos a causa de la infracción, permanencia o transitoriedad de los riesgos y reincidencia por comisión en el término de un año de más de una infracción de la misma naturaleza cuando así haya sido declarado por resolución firme.

${ }^{54}$ Para las infracciones leves: grado mínimo, hasta 6.000 euros, grado medio: desde 6.001 a 18.000 euros, grado máximo: desde 18.001 a 30.000 euros. Para las infracciones graves, grado mínimo: desde 30.001 a 60.000 euros, grado medio: desde 60.001 a 78.000 euros, grado máximo: Desde 78.001 a 90.000 euros. Para las infracciones muy graves: grado mínimo: desde 90.001 a 300.000 euros, grado medio: desde 300.001 a 600.000 euros, grado máximo: desde 600.001 a 1.000 .000 de euros, pudiendo rebasar dicha cantidad hasta alcanzar el quíntuplo del valor de los productos o servicios objeto de la infracción.

55 Véase, Ley Orgánica 15/2003, de 25 de noviembre, por la que se modifica la Ley Orgánica 10/1995, de 23 de noviembre, del Código Penal. BOE núm. 283, de 26 de noviembre de 2003. Artículo 100, que modifica el artículo 282, establece que «serán castigados con la pena de prisión de seis meses a un año o multa de 12 a 24 meses los fabricantes o comerciantes que, en sus ofertas o publicidad de productos o servicios, hagan alegaciones falsas o manifiesten características inciertas sobre los mismos, de modo que puedan causar un perjuicio grave y manifiesto a los consumidores, sin perjuicio de la pena que corresponda aplicar por la comisión de otros delitos».
} 
seis a doce meses e inhabilitación especial para profesión, oficio, industria o comercio por tiempo de tres a seis años los productores, distribuidores o comerciantes ${ }^{56}$.

\section{CONCLUSIÓN.}

La publicidad en términos generales puede influir en la capacidad de elección de la sociedad. Cuando se trata de publicidad sobre medicamentos la importancia es mayor, puesto que pueden originar daños en la salud pública. Por ello la regulación a la que esta sometida es de gran relevancia.

La publicidad en general viene regulada mediante la Ley 34/1988, de 11 de noviembre, General de Publicidad ${ }^{57}$. Sin embargo, esta remite a la regulación específica para la legislación en materia de publicidad de medicamentos.

Existen diversas normas que hacen referencia a la publicidad de medicamentos, tanto en el ámbito comunitario como en el nacional. En España, la principal norma en la que se regula la publicidad de medicamentos es en el Real Decreto 1416/1994, de 25 de junio, por el que se regula la publicidad de los medicamentos de uso humano ${ }^{58}$. No cabe duda de que en el Real Decreto Legislativo 1/2015, de 24 de julio, por el que se aprueba el texto refundido de la Ley de garantías y uso racional de los medicamentos y productos sanitarios $^{59}$ también se realizan especificaciones relevantes, concretamente en su artículo 80 en la materia objeto de estudio.

Para que la publicidad sea legal y se adecúe a la normativa vigente, existen diferentes métodos de control, además, la publicidad en materia de aquellos medicamentos los cuáles tienen un elevado riesgo para la salud en caso de uso inadecuado, tendrán que haber sido aprobada su publicidad por parte de las Administraciones.

Es cierto que las empresas responsables de la comercialización son las que deben realizar una publicidad adecuada, pero, para facilitar esta acción y en caso de duda puedan recurrir a asociaciones que velan por una publicidad responsable (controles internos). Sin embargo, se realizan también controles externos a la empresa ejercidos por órganos jurisdiccionales y administrativos.

Por último, en caso de que la publicidad no sea conforme a la normativa vigente, las infracciones tipificadas en materia de publicidad serán diferentes en caso de que se trate

\footnotetext{
${ }^{56}$ Véase, ley Orgánica 1/2015, de 30 de marzo, por la que se modifica la Ley Orgánica 10/1995, de 23 de noviembre, del Código Penal. BOE núm. 77, de 31 de marzo de 2015. Artículo 190, por el que se añade el artículo 362 bis del Código Penal, según el cuál "serán castigados con la pena de prisión de uno a cuatro años, multa de seis a doce meses e inhabilitación especial para profesión, oficio, industria o comercio por tiempo de tres a seis años los productores, distribuidores o comerciantes que pongan en peligro la salud de los consumidores elaborando productos cuyo uso no se halle autorizado y sea perjudicial para la salud, o comerciando con ellos".

${ }^{57}$ BOE núm. 274 de 15 de noviembre de 1988.

${ }^{58}$ Véase, artículo 10. BOE núm. 180, de 29 de julio de 1994.

59 BOE núm. 177, de 25 de julio de 2015.
} 
de una publicidad inadecuada de un medicamento autorizado o, en su caso, el medicamento no estuviese autorizado o resultase ser perjudicial.

\section{REFERENCIAS}

Bombillar Sáenz, F. M. (2010) Intervención administrativa y régimen jurídico del medicamento en la Unión Europea. Tesis Doctoral. Granada: Ed. Universidad de Granada.

España. (1986, 29 de abril) Ley 14/1986, de 26 de abril, General de Sanidad. Boletín Oficial del Estado no. 102.

España. (2009, 31 de diciembre) Ley 29/2009, de 30 de diciembre, por la que se modifica el régimen legal de la competencia desleal y de la publicidad para la mejora de la protección de los consumidores y usuarios. Boletín Oficial del Estado no. 315.

España. (1991, 11 de enero) Ley 3/1991, de 10 de enero, de Competencia Desleal. Boletín Oficial del Estado no. 10.

España. (1988, 15 de noviembre). Ley 34/1988, de 11 de noviembre, General de Publicidad. Boletín Oficial del Estado no. 274.

España. (2015, 31 de marzo). Ley Orgánica 1/2015, de 30 de marzo, por la que se modifica la Ley Orgánica 10/1995, de 23 de noviembre, del Código Penal. Boletín Oficial del Estado no. 77.

España. (1985, 18 de diciembre) Orden de 10 de diciembre de 1985, del Ministerio de Sanidad y Consumo, por la que se regulan los mensajes publicitarios referidos a medicamentos y determinados productos sanitarios. Boletín Oficial del Estado no. 302.

España. (1994, 29 de julio) Real Decreto 1416/1994, de 25 de junio, por el que se regula la publicidad de medicamentos de uso humano. Boletín Oficial del Estado no. 180.

España. (2015, 25 de julio) Real Decreto Legislativo 1/2015, de 24 de julio, por el que se aprueba el texto refundido de la Ley de garantías y uso racional de los medicamentos y productos sanitarios. Boletín Oficial del Estado no. 177.

García Vidal, Á. (2013) La promoción de medicamentos dirigida a profesionales sanitarios. Estudio desde la perspectiva del Derecho Mercantil. Madrid; Marcial Pons. 271 págs.

Ministerio de Sanidad, Consumo y Bienestar Social. (2019) Guía para la publicidad de medicamentos de uso humano dirigida al público, cit. p. 5. 
Sánchez Ruiz, M. M. (2017) Capítulo noveno. Algunas cuestiones jurídicas sobre la publicidad de los medicamentos en Cuestiones actuales de la prestación farmacéutica y los medicamentos. Mํㅡㄹ Belén García Romero y Mํㅡㄹ Del Mar de la Peña Amorós. ISBN: 978-84-9148-487-5.

Sarrato Martínez, L. (2015): Régimen jurídico-administrativo del medicamento. La Ley. Las Rozas; Madrid.

Unión Europea. (2001, 6 de noviembre) Directiva 2001/83/CE, del Parlamento Europeo y del Consejo, por la que se establece un código comunitario sobre medicamentos de uso humano. Diario Oficial de la Unión Europea. Ley 311.

Unión Europea. (2006, 12 de diciembre) Directiva 2006/114/CE, del Parlamento Europeo y del Consejo, sobre publicidad engañosa y publicidad comparativa. Diario Oficial de la Unión Europea. Ley 376.

Unión Europea. (2010, 10 de marzo) Directiva 2010/13/UE, del Parlamento Europeo y del Consejo, sobre la coordinación de determinadas disposiciones legales, reglamentarias y administrativas de los Estados miembros relativas a la prestación de servicios de comunicación audiovisual. Diario Oficial de la Unión Europea. Ley 75.

Vida Fernández, J. (2015) Concepto y régimen jurídico de los medicamentos. Su distinción con otros productos para el cuidado de la salud. Valencia; Ed. Tirant lo Blanch.

Vida Fernández, J. y Faus Santasusana, J. (2017) Tratado de Derecho Farmacéutico. Cizur Menor. Navarra; Ed. Thomson Reuters-Aranzadi.

\section{AUTORES}

\section{Carlos del Castillo Rodríguez}

Licenciado en Farmacia, Universidad Complutense de Madrid; Licenciado en Derecho, Universidad Nacional de Educación a Distancia; Doctor Europeo, Università Alma Mater Studiorum di Bologna; Profesor Contratado Doctor de Universidad. Departamento de Farmacia Galénica y Tecnología Alimentaria (Historia de la Farmacia y Legislación Farmacéutica).

carlosdelcastillo@farm.ucm.es

Orcid ID: https://orcid.org/0000-0003-2247-5966

GoogleScholar: https://scholar.google.es/citations?hl=es\&user=b br7n8AAAAJ

\section{Silvia Enríquez Fernández}

Graduada en Farmacia, Universidad Complutense de Madrid; Investigadora doctoral de la Universidad Complutense de Madrid. Departamento de Farmacia Galénica y Tecnología Alimentaria (Historia de la Farmacia y Legislación Farmacéutica).

Contacto: silvienr@ucm.es. 
Sobre la publicidad de los medicamentos: legislación

Orcid ID: https://orcid.org/0000-0002-9868-1312

Google Scholar: https://scholar.google.es/citations?hl=es\&user=TeD79asAAAAJ 